\title{
FUNÇÃO ENDÓCRINA, HOMEOSTASE DA GLICOSE E RESISTÊNCIA À INSULINA APÓS TRANSPLANTE DE PÂNCREAS
}

\author{
Endocrine Function, Glucose Homeostasis and After Pancreas Transplantation Insulin Resistance
}

\author{
Marcelo Junges Hartmann; Marcello Casaccia Bertoluci; Maria Cláudia Guterres; Luciano Passamini Diogo; David Saitovitch; \\ Salvador Gullo Neto; Manlio Falavigna; Leonardo Viliano Kroth; Moacir Alexandre Traesel
}

\section{RESUMO}

O objetivo do transplante de pâncreas é o restabelecimento da homeostase da glicose para prevenir o aparecimento ou a progressão de complicações crônicas do diabetes e melhorar a qualidade de vida. Transplantados com sucesso apresentam melhora significativa no controle metabólico, que pode ou não estar associado à hiperinsulinemia e ao aumento da resistência insulínica. O HOMA (Homeostasis Model Assessment) pode ser útil para avaliar a função das células beta pós-transplante.

Descritores: Transplante de Pâncreas, Resistência à Insulina, Ilhotas Pancreáticas

\section{Instituição:}

Hospital São Lucas da PUCRS - Porto Alegre - RS

${ }^{*}$ Hospital de Clínicas de Porto Alegre - RS

\section{Correspondência:}

Marcelo Junges Hartmann

Avenida Ipiranga, 6690 conjunto 620, Jardim Botânico, Porto Alegre, RS CEP: $90610-000$

Fone/FAX: 51 3336-0304

E-mail: marcelohartmann@hotmail.com

\section{INTRODUÇÃO}

O diabetes mellitus (DM) é uma patologia muito freqüente, que não pode ser curada pela insulina exógena. O transplante de pâncreas é a única terapia disponível capaz de restabelecer a secreção endógena de insulina que geralmente resulta em um estado de euglicemia.

Muitos pacientes portadores de DM tipo 1 (DM1) acabam desenvolvendo complicações crônicas como nefropatia, retinopatia e neuropatia, com sérios prejuízos relacionados à qualidade de vida. O DCCT (Diabetes Control and Complication Trial) demonstrou que o controle glicêmico pode limitar a taxa de progressão das complicações do DM1. Entretanto, o tratamento intensivo pode determinar um risco três vezes maior do paciente desenvolver hipoglicemia severa. ${ }^{1}$

Desde o primeiro transplante pancreático realizado por Kelly e Lillehei em $1966{ }^{2}$ muitos avanços ocorreram, especialmente os relacionados à técnica cirúrgica, imunossupressão e aos cuidados pós-operatórios, com uma melhora progressiva dos resultados do transplante ${ }^{3,4,5}$ e conseqüente aumento do número de casos realizados em todo o mundo. Até o final de 2004, mais de 23.000 transplantes haviam sido notificados no Registro Internacional de Transplante de Pâncreas ${ }^{7}$

A sobrevida do paciente um ano após ser submetido ao transplante de pâncreas é superior a 95\% nos Estados Unidos. A taxa de sobrevida do enxerto pancreático no primeiro ano (paciente livre do uso de insulina) em transplante conjugado rim/pâncreas é de $85 \%$; em pacientes que recebem pâncreas e já têm um rim transplantado é de $78 \%$, e em transplante isolado de pâncreas é de cerca de $76 \%$ ?

O objetivo do transplante de pâncreas é o restabelecimento da homeostase da glicose, para prevenir o aparecimento ou a progressão das complicações crônicas do diabetes e melhorar a qualidade de 
vida dos pacientes. Vários trabalhos têm demonstrado os efeitos benéficos do transplante, no que diz respeito às complicações crônicas do DM. Há evidências de que o transplante pancreático está associado com a regressão histológica da nefropatia diabética ${ }^{8-10}$, melhora na condução nervosa sensitiva e motora ${ }^{11,12}$ e aumento na expectativa de vida de pacientes com neuropatia autonômica. ${ }^{13}$

Estudos que avaliaram a qualidade de vida demonstraram benefícios consistentes, como retorno ao trabalho e gestações. ${ }^{14,15}$

Um estudo observacional comparou a sobrevida de pacientes diabéticos dez anos após terem sido submetidos a transplante simultâneo de pâncreas e rim com pacientes que receberam apenas rim. Houve mortalidade de $80 \%$ no grupo de controle e $20 \%$ no grupo estudado, com uma significativa melhora no controle metabólico do grupo com transplante simultâneo. ${ }^{16}$

Já existem algumas evidências sugerindo um possível benefício do transplante pâncreas-rim na doença macrovascular, como por exemplo, redução da espessura da camada íntima da carótida. ${ }^{17-20}$

Em relação à evolução da retinopatia diabética pós-transplante de pâncreas, ainda não há consenso na literatura. Desde o primeiro relato de benefício do transplante pancreático na retinopatia diabética (Ramsay et al 1988 - Universidade de Minnesota), ${ }^{21}$ resultados conflitantes têm sido publicados. Entretanto, a maioria dos estudos apresenta limitações pelo curto período de acompanhamento e pela inclusão de pacientes com retinopatia avançada. O DCCT demonstrou que o tratamento intensivo está associado a um efeito benéfico em todos os níveis de retinopatia estudados, tendo sido observado somente após três anos de tratamento intensivo. ${ }^{22,23}$ É importante ressaltar que foram excluídos do estudo pacientes com retinopatia proliferativa severa, patologia usualmente presente nos pacientes transplantados de pâncreas.

Em um estudo com período de observação de 40 meses póstransplante simultâneo de pâncreas e rim, observou-se regressão da retinopatia em 9\%, estabilização em 73\% e progressão em 18\% dos receptores, enquanto que no grupo transplantado com perda do enxerto pancreático, 54\% estabilizaram-se e 46\% progrediram. ${ }^{24}$

\section{CONTROLE METABÓLICO}

Uma importante melhora documentada nos pacientes submetidos com sucesso a transplante de pâncreas é o controle metabólico. Esses pacientes apresentam hemoglobina glicosilada, glicemia de jejum e pós-prandial mais próximos da normalidade, quando comparados com seus controles. ${ }^{25,26}$ Essa melhora tem sido relatada inclusive nos estudos a longo prazo, com manutenção da capacidade de secreção de insulina, controle glicêmico adequado e restauração da contra-regulação hipoglicêmica. ${ }^{27-31}$

Entretanto, mesmo nos transplantes com sucesso inicial, excelente controle metabólico e insulino-independência, há pacientes que apresentam episódios de hiperglicemia, além de outros que, ao longo de seus seguimentos, necessitam retornar ao uso da insulina exógena. Isso pode acontecer devido a vários mecanismos, como desenvolvimento de resistência tecidual à ação da insulina, recorrência da doença auto-imune, rejeição aguda ou crônica, ou até mesmo efeito tóxico das drogas imunossupressoras. ${ }^{32-35}$

Classicamente, tem sido descrito que o transplante de pâncreas restaura o estado euglicêmico nos diabéticos insulino-dependentes, à custa de uma significativa hiperinsulinemia periférica conseqüente à drenagem venosa sistêmica do enxerto pancreático. Porém, estudos mais recentes avaliando possíveis vantagens metabólicas da drenagem venosa portal à drenagem sistêmica não têm demonstrado diferenças importantes do ponto de vista endocrinológico. ${ }^{36,37}$

Além desses fatores que podem influenciar o controle glicêmico, diferentemente do pâncreas nativo, o pâncreas transplantado é um órgão denervado, que pode estar associado a uma diminuição do “feedback” negativo da secreção de insulina. ${ }^{38,39}$

Geralmente, no seguimento de pacientes transplantados de pâncreas há uma preocupação com o controle glicêmico e a monitoração de rejeição. Entretanto, não há uma rotina para o diagnóstico e a monitoração da ocorrência de hiperinsulinemia ou avaliação adequada de resistência insulínica.

\section{RESISTÊNCIA À INSULINA}

A resistência à insulina (RI) é uma anormalidade metabólica característica de indivíduos com diabetes tipo 2, diabetes tipo 1 descontrolado, cetoacidose diabética e obesidade. O processo de envelhecimento também está relacionado à progressão da RI. Em populações normais, a RI ocorre em 20 a $25 \%$ dos indivíduos. Em populações de não-diabéticos, a redução da ação insulínica pode estar acompanhada de um grupo de alterações metabólicas e cardiovasculares que compreende hipertensão arterial, hipertrigliceridemia, redução do HDL-colesterol, intolerância aos carboidratos, obesidade centrípeta, aumento do inibidor-1 do ativador do plasminogênio, hiperuricemia e doença cardiovascular aterosclerótica. Esse conjunto de alterações da RI é conhecido como síndrome de resistência à insulina ou síndrome metabólica. ${ }^{40,41}$

Tanto o controle glicêmico quanto a RI podem ser influenciados pelo uso de drogas imunossupressoras utilizadas após o transplante pancreático, que são potencialmente diabetogênicas. Os corticosteróides estão associados ao aumento da RI e da gliconeogênese, além da exacerbação da lesão das ilhotas, desencadeada pelos inibidores da calcineurina no pâncreas transplantado. ${ }^{42-44} \mathrm{O}$ desenvolvimento de RI não é raro nas primeiras semanas seguintes ao transplante. Geralmente, responde à redução da dose de corticosteróide e/ou do inibidor da calcineurina. ${ }^{45-48}$

A avaliação da RI pode ser obtida utilizando-se a técnica de clamp, descrita por DeFronzo e colaboradores em 1979, como método para quantificar a secreção de insulina e a resistência à sua ação. É considerado o método de referência para uma aferição acurada da sensibilidade à insulina in vivo. Entretanto, esse método é caro, laborioso, demanda tempo e não é disponível em larga escala, especialmente para estudos populacionais. ${ }^{49,50}$

Mathews e colaboradores desenvolveram um modelo matemático que prediz a sensibilidade à insulina pela simples medida da glicemia e insulinemia de jejum. Esse método foi chamado de HOMA (Homeostasis Model Assessment) e dele se extraem os índices HOMA-IR, HOMA-Beta e HOMA-IS, que visam traduzir a sensibilidade à insulina e a capacidade de secreção da célula beta. Eles se basearam em dados da literatura para construir curvas relacionando glicemia (no estado de homeostasia) à resposta insulínica em indivíduos saudáveis e com graus variados de anormalidade no metabolismo da glicose. ${ }^{51}$

O modelo prediz uma insulinemia e glicemia para uma dada sensibilidade tecidual e capacidade de secreção de insulina. Inversamente, se conhecidas simultaneamente a glicemia e a insulinemia, o modelo pode fornecer os índices HOMA-IR, HOMABeta e HOMA-IS pelas seguintes equações: 
HOMA-IR = Glicemia $(\mathrm{mMol} / \mathrm{l}) \mathrm{x}$ Insulinemia $(\mathrm{mU} / \mathrm{l}) \div 22,5$

HOMA-Beta $=20 \times$ Insulinemia $(\mathrm{mU} / \mathrm{l}) /($ Glicemia $(\mathrm{mMol} / \mathrm{l})$ - 3,5)

HOMA-IS = 1/HOMA-IR

Os autores encontraram uma correlação positiva e altamente significativa entre a RI avaliada pelo Homa e pelo clamp $(\mathrm{r}=0,88$, $\mathrm{p}<0,0001)^{51,52}$

O HOMA, assim como outros índices de resistência à insulina, já foi validado em populações de transplantados renais. ${ }^{53}$

Aguilera e colaboradores realizaram o primeiro estudo que demonstrou ser o HOMA um teste válido para avaliar a secreção de insulina em pacientes transplantados de pâncreas e rim. Segundo esses autores, a informação obtida através do HOMA é similar àquela do teste de tolerância à glicose oral (TTGO). O modelo do HOMA utiliza a função da célula beta e a resposta hepática e periférica para analisar a interação entre insulina e glicose séricas. A drenagem venosa não portal dos transplantes de pâncreas poderia ser uma crítica à utilização do HOMA nesses pacientes pela participação hepática importante na construção desse modelo. Entretanto, a correlação positiva observada nesse estudo entre os níveis médios do HOMA-Beta e os níveis médios da área sob curva obtidos pelo TTGO sugere que isso não seja um problema metodológico relevante. A maioria dos transplantados apresentou normoglicemias, HOMA-Beta mais altos, possivelmente pela drenagem venosa sistêmica e HOMA-IS mais baixos provavelmente pela resistência à insulina induzida pela corticoterapia crônica. ${ }^{54}$

Há evidências, inclusive, de que a tolerância à glicose oral e a secreção de insulina estimulada (após o TTGO) possam ser previsores* da função endócrina do enxerto pancreático em longo prazo, nos estudos com mais de dez anos de seguimento. ${ }^{55}$

Entretanto, há poucos relatos na literatura sobre a resposta insulínica (curva de insulina) após sobrecarga de glicose em transplantados de pâncreas.
Em um estudo transversal realizado no Hospital São Lucas da PUCRS (manuscrito enviado para a publicação) que avaliou uma amostra de 30 pacientes transplantados de pâncreas com drenagem venosa sistêmica, verificou-se a ocorrência de insulino-resistência em dois terços desses pacientes, de acordo com os critérios definidos pelo Brazilian Metabolic Syndrome Study (BRAMS) ${ }^{56} \mathrm{O}$ controle glicêmico não foi influenciado pelo aumento de RI. Esses pacientes apresentaram HOMA-Beta mais alto quando comparados com transplantados com RI normal, evidenciando-se a capacidade do pâncreas transplantado de sobrepor-se ao aumento da RI. Além disso, um subgrupo de transplantados apresentou RI e insulinemia normais, o que sugere que outros fatores podem estar envolvidos na hiperinsulinemia observada por outros autores em transplantes pancreáticos com drenagem venosa sistêmica.

\section{CONCLUSÃO}

O transplante de pâncreas é uma alternativa terapêutica segura para pacientes diabéticos complicados. Transplantados com sucesso apresentam melhora significativa do controle metabólico.

A longo prazo, no pós-transplante de pâncreas, a função do enxerto pode sofrer alterações como disfunção e até mesmo, recidiva do diabetes. Por isso, é importante que seja avaliada a função das células beta no seguimento desses pacientes. Geralmente, isso é feito levando-se em conta a insulino-independência, a secreção de peptídeo $\mathrm{C}$ e o controle glicêmico, medidas essas consideradas interdependentes e determinantes da função das células beta.

O HOMA pode ser útil para essa avaliação, pois fornece uma estimativa da capacidade de secreção das células beta e da resistência à insulina - além de ser um método mais fácil, rápido e de custo inferior, que não requer dieta especial, em comparação com outros métodos como clamp ou TTGO.

* Essa palavra não existe. Pode-se trocar por “previsores”. (N.R.)

\section{ABSTRACT}

The goal of the pancreatic transplantation is to restore the glucose homeostasis to prevent or improve secondary complications to diabetes and improving the quality of life. Successful transplantations have a good metabolic controlling. These might be or not associated to hyperinsulinemia and increased insulin resistance. HOMA may be useful in evaluating post-transplant beta-cell function.

Keywords: Pancreas Transplantation, Insulin Resistance, Islets of Langerhans

\section{REFERÊNCIAS}

1. Diabetes Control and Complications Trial Research Group. The effect of intensive treatment of diabetes on the development and progression of long- term complications in insulin- dependent diabetes mellitus. N. Engl J Méd. 1993;329:977-86.

2. Kelly W, Lillehei R, Merkel F, Idezuki Y, Goetz F. Allotransplantation of the pancreas and duodenum along with the kidney in diabetic nephropathy. Surgery 1967;61(6):827-37.

3. Sutherland D, Gruessner R, Dunn D, Matas A, Humar A, Kandaswamy R, et al. Lessons learned from more than 1000 pancreas transplants at a single institution. Ann Surg. 2001;4:463-501.
4. Sollinger H, Odorico J, Knechtle S, D' Alessandro A, et al. Experience with 500 simultaneous pancreas- kidney transplants. Ann Surg. 1998;228:284-96.

5. Stratta R, TaylorR, Bynon S, Lowel J, et al. Surgical treatment of diabetes mellitus with pancreas transplantation. Ann Surg. 1994;220(6):809-17.

6. Odorico J, Sollinger H. Technical and immunosupressive advances in transplantation for insulin-dependent diabetes mellitus.World J Surg. 2002;26:194-211.

7. Gruessner AC, Sutherland DE. Pancreas transplant outcomes for United States (US) and non-US cases as reported to the United Network for Organ Sharing UNOS) and the International Pancreas Transplant Registry (IPTR) as of June 2004. Clin Trasnplant. 2005;19(4):433-55. 
8. Bilous RW, Mauer SM, Sutherland DER, Najarian JS, Goetz FC, Steffes MW. The effects of pancreas transplantation on the glomerular structure of renal allograft in patients with insulin-dependent diabetes. New England Journal of Diabets. 1989;1989(321):80-5.

9. Bohman S-O, Tydén G, Wilczek H, Ostman J, Llundgren G, Jaremko G, et al. Prevention of kidney Graft Diabetic Nefrophaty by Pancreas Transplantation in Man. Diabetes. 1985;34(306-308).

10. Fioreto P, Steffes MW, Sutherland DER, Goetz FC, Mauer M. Reversal of lesion of diabetic nephopathy after pancreas transplantation. New England of Medicine. 1988;33:69-75.

11. Allen RD, Al-Harbi IS, Morris JG, Clouston PD, O'Connel PJ, Charpman JR, et al. Diabetic Neurophaty after Pancreas Transplantation. Transplantation. 1997;63:830-8.

12. Navarro X, Sutherland DER, Kennedy WR. Long-effects of pancreatic transplantationon diabetes neurophaty. Annals of Neurology. 1997;42:727-36.

13. Navarro X, Kennedy WR, Loewenson RB, Sutherland DER. Influence of pancras transplantation on cardiorrespiratory reflexes, nerve condution, and mortality in diabetes mellitus. Diabetes. 1990;39:802-6.

14. Barrou B, Baldi A, Bitker MO, Squifflet JP, Grussner RW, Sutherland DER. Pregnancy after pancreas transplantation: report of four new cases and review of literature. Transplant Proc. 1995;27:3043-4.

15. Zehrer CL, Gross CR. Comparation of quality of life between pancreas /kidney and kidney transplant recipients:one-year follow-up. Transplant Proc. 1994;26:508-9.

16. Tydén G, Bolinder J, Solders G, Brattström C, Tibell A, Groth C-G. Improved Survival in Patients with Insulin-Dependent Diabetes Mellitus and End-Stage Diabetic nephropathy 10 year after Combined Pancreas and Kidney Transplantation. Transplantation. 1999;67(5):645-8.

17. Jukema JW, Smets YFC, Fijter JWd. Impact of Simultaneos Pancreas and Pancreas and Kidney Transplantation on Progression of Coronary Atherosclerosis in Patients With End-Stage Renal Failure due to Type 1Diabetes. Diabetes Care. 2002;25(2):906-11.

18. La Rocca E, Fiorina P, Di Carlo V, Astorri E, Rossetti C, Lucigniane G, Fazio F, Giudici D, Cristallo M, Bianchi G, Pozza G, Secchi A. Cardiovascular outcomes after kidney-pancreas and kidney-alone transplantation. Kidney Int. 2001;60(5):1964-71.

19. Biesenbach G, Konigsrainer A, Gross C, Margreiter R. Progression of macrovascular diseases is reduced in type 1 diabetic patients after more than 5 years successful combined pancreas-kidney transplantation in comparison to kidney transplantation alone. Transplant Int. 2005;18(9):1054-60

20. Larsen JL, Colling CW, Ratanasuwan T, Burkman TW, Lynch TG, Erickson JM, et al. Pancreas Transplantation Improves Vascular Disease in Pacients With Type 1 Diabetes. Diabetes Care. 2004;27(7):1706-11

21. Ramsay RC, Goetz FC, Sutherland DE, et al. Progression of diabetic retinopathy after pancreas transplantation for insulin-dependent diabetes mellitus. N Engl J Med. 1988;318:208-14.

22. Diabetes Control and Complications Trial Research Group. The effect of intensive diabetes treatment on the progression of diabetic retinopathy in insulin-dependent diabetes mellitus. Arch Ophthalmol. 1995;113:36-49.

23. Diabetes Control and Complications Trial Research Group. Progression of retinopathy with intensive versus conventional treatment in the Diabetes Control and Complications Trial. Ophthalmology. 1995;102:647-61

24. Königsreiner A, Miller K, Steurer W, et al. Does pancreas transplantation influence the course of diabetic retinopathy? Diabetologia. 1991;34:86-8.

25. Balsells MF, Esmatjes E, Maria JR, Casamitjana R, Astudillo E, Cruz LF. Successful Pancreas and Kidney Transplantation: A view of Metabolic Control. Clinical Transplantation. 1998;12:582-7.

26. Cottrell DA. Normalization of Insulin Sensitivity and Glucose Homeostasis in Type I Diabetic Pancreas Transplant Recipients: Journal of Clinical Endocrinology and Metabolism. 1996;81(10):3513-9.
27. Robertson RP, Kendal D, Teuscher A, Sutherland DER. Long-term metabolic control with pancreatic transplantation. Transplant Proc. 1994;26(2):386-7.

28. Tajra LC, Martin X, Benchaid M, Dawara M, Lefrancois N, Dubernard JM. Longterm metabolic control in pancreas transplant patients according to 3 techniques. Transpl Proc. 1998,30:268-9.

29. Tyden G, Bolinder J, Brattstrom C, Tibell A, Groth CG. Long term metabolic control in recipients of segmental or whole- organ pancreatis grafts with enteric exocrine diversion and funtion beyond 5 years. Transpl Proc. 1998,30:634.

30. Hopt UT, Drognitz O. Pancreas organ transplantation. Short and long- term results in terms of diabetes control. Langenbecks Arch Surg. 2000;385(6):379-89.

31. Paty BW, Lanz K, Kendall DM, Sutherland DE, Robertson RP. Restored hypoglycemic counterregulation is stable in successfull pancreas transplant recipients for up to 19 years after transplantation. Transplantation. 2001;72(6):1103-7.

32. Jones JW, Mizrahi SS, Bentley FR: Type II biabetes after combined kidney and pancreas transplantation for type I diabetes mellitus and end- stage renal desease. Clin Transplant. 1996;10:574-5.

33. Tyden G, Reinholt FP, Sundkvist G, Bolinder J. Recurrence of autoimmune diabetes mellitus in recipients of cadaveric pancreatic grafts. N Engl J Méd. 1996;335:860-3.

34. Petruzzo P, Andreelli F, McGregor B, Lefrancois N, Dawara M, Feitosa LC, Dubernard JM, et al. Evidence of recurrence type I diabetes following HLAmismatched pancreas transplantation. Diabetes Metab. 2000;26(3):215-8.

35. Egidi FM. Management of hyperglycaemia after pancreas transplantation: are new immunosupressants the answer? Drugs. 2005;65(2):153-66.

36. Martin X, Petruzzo P, Dawahra M, Feitosa Tajra LC, Da Silva M, Pibiri L, Chapuis F, Dubernard JM, Lefrancois N. Effects of portal versus systemic venous drainage in kidney-pancreas recipients. Transpl Int. 2000;13(1):64-8.

37. Alonso A, Fernandez C, Cillero S, Gomez M, Aguirrezabalaga J, Valdes F. Effects of portal versus systemic venous drainage in pancreas and kidney-pancreas transplantation. Transplant Proc. 2007;39(7):2335-7.

38. Luzi L, Battezzati A, Perseghin G, et al. Lack of feedback inhibition of insulin secretion in denervated human pancreas. Diabetes. 1992;41:1632.

39. Nankivel BJ, Chapman JR, Bovington KJ, Spicer ST, et al. Clinical determinants of glucose homeostasis after pancreas transplantation. Transplantation. 1996;61(12):1705-11.

40. Geloneze B, Tambascia MA. Avaliação laboratorial e diagnóstico da resistência insulínica. Arq Bras Endocrinol Metab. 2006;Abr 50(2):208-15.

41. Hanley AJG, Willians K, Stern MP, Haffner S. Homeostasis Model Assessment of Insulin Resistance in Relation to the Incidence of Cardiovascular Disease - The San Antonio Heart Study. Diabetes Care. 2002;25:1177-84.

42. Boots JM, van Duijnhoven EM, Christiaans MH, Wolffenbuttel BH, van Hooff JP. Glucose metabolism in renal transplant recipients on tacrolimus: the effect of steroid withdrawal and tacrolimus trough level reduction. J Am Soc Nephrol. 2002;13(1):221-7.

43. van Hoof JP, Christiaans MH, van Duijnhoven EM. Evaluating mechanisms of posttransplant diabetes mellitus. Nephrol Dial Transplant. 2004;19(Suppl 6): vi8-vi12.

44. van Duijnhoven EM, Christiaans MH, Boots JM, Nieman FH, Wolffenbuttel BH, van Hooff JP. Glucose metabolism in the first 3 years after renal transplantation in patients receiving tacrolimus versus cyclosporine-based immunosupression. J Am Soc Nephrol. 2002;13(1):213-20.

45. Midtvedt K, Hjelmesaeth J, Hartmann A, Lund K, Paulsen D, Egeland T, Jenssen T. Insulin resistance after renal transplantation: the effect of steroid dose reduction and withdrawal. J Am Soc Nephrol. 2004;15(12):3233-9.

46. Drachenberg CB, Klassen DK, Weir MR, et al. Islet cell damage associated with tacrolimus and cyclosporine: morphological features in pancreas allograft biopsies and clinical correlation. Transplantation. 1999;68(3):396-402. 
47. Humar A, Parr E, Drangstveit MB, Kandaswamy R, Gruessner AC, Sutherland DER. Steroid withdrawal in pancreas transplant recipients. Clin Transplant. 2000; $14: 75-8$

48. Kaufman DB, Leventhal J, Koffron AJ, et al. A prospective study of rapid corticosteroid elimination in simultaneous pancreas-kidney transplantation: comparison of two maintenance immunossupression protocols: tacrolimus/ mycophenolate mofetil versus tacrolimus/ sirolimus. Transplantation. 2002;73(2):169-77.

49. DeFronzo RA, Tobin JD, Andres R. Glucose clamp technique: a method for quantifying insulin secretion and resistance. Am J Physiol. 1979;237:E214-E223.

50. Bonora E, Targher G, Alberiche M, Bonadonna RC et al. Homeostasis Model Assessment Closely Mirrors the Glucose Clamp Technique in the Assessment of Insulin Sensitivity. Diabetes Care. 2000;23(1):57-63.

51. Mathews DR, Hosker JP, Rudenski AS, Naylor BA, Treacher DF, Turner RC. Homeostasis model assessment: insulin resistance and beta-cell function from fasting plasma glucose and insulin concentrations in man. Diabetologia. 1985;28:412-9.
52. Wallace TM, Levy JC, Mathews DR. Use and Abuse of HOMA Modeling. Diabetes Care. 2004;27:1487-95.

53. Oterdoon LH, de Vries APJ, van Son WJ et al. Validation of Insulin Resistance Indexes in a Stable Renal Transplant Population. Diabetes Care. 2005;8:2424-9.

54. Aguilera E, Recasens, M, Flores L, Ricart HJ, Casamitjana R, Fernández-Cruz L, Esmatjes E. HOMA Test in Diabetic Patients With Simultaneous Pancreas and Kidney Transplantation. Transplant Proc. 2002;34:206-8.

55. Pfeffer F, Nauck MA,Drognitz O, Benz S,von Dobschuetz E, Hopt UT. Postoperative oral glucose tolerance and stimulated insulin secretion: a predictor of endocrine graft function more than 10 years after pancreas-kidney transplantation. Transplantation. 2003;76(10):1427-31.

56. Geloneze B, Geloneze S, Ermetice M, Murro A, Lima M, Astiarraga B, Tambascia M. Obesity and glucose tolerance status are major determinants of the prevalence of insulin resistance in an admixtured population. Brazilian Metabolic Syndrome Study (BRAMS). Diabetic Medicine. 2006;23(Suppl. 4):608-753. 